\title{
EFEITO DO DIÂMETRO FOLICULAR SOBRE A QUALIDADE DOS OÓCITOS OBTIDOS DE OVÁRIOS DE OVELHAS (Ovis aries) E CABRAS (Capra hircus)
}

\author{
Ricardo de Macêdo Chaves, ${ }^{1,2}$ Cristiano Aguiar Filho, ${ }^{2}$ Edivaldo Rosas Santos Júnior, ${ }^{2}$ \\ José Monteiro Almeida Filho, ${ }^{1}$ Paulo Fernandes de Lima ${ }^{2}$ e Marcos Antônio Lemos de Oliveira ${ }^{2}$ \\ 1. Laboratório de Reprodução Animal da Escola de Medicina Veterinária da Universidade Estadual do Maranhão \\ E-mail: rmchaves@hotmail.com \\ 2. Laboratório de Biotécnicas Aplicadas à Reprodução do Departamento de Medicina Veterinária da Universidade Federal Rural de Pernambuco
}

\section{RESUMO}

O presente estudo teve como objetivo avaliar a influência do diâmetro folicular sobre a qualidade dos oócitos obtidos de ovários de fêmeas ovinas e caprinas. Utilizaram-se 156 ovários de ovelhas e 105 de cabras, provenientes de abatedouro. Os folículos foram mensurados, aspirados e divididos em três classes de diâmetro folicular: classe $1(\mathrm{CI})-2$ a $3 \mathrm{~mm}$, classe $2(\mathrm{CII})-4$ a $5 \mathrm{~mm}$ e classe 3 (CIII) $-6 \mathrm{~mm}$. Os oócitos recuperados foram avaliados e classificados quanto ao aspecto morfológico em cinco grupos de qualidade: grau 1 (GI), grau 2 (GII), grau 3 (GIII), desnudo (D) e atrésicos (A). Dos 468 folículos aspirados de ovinos, foram encon- trados 327 CI, 84 CII, 57 CIII e 83 GI, 78 GII, 95 GIII, 119 D, 93 A. E dos 422 folículos aspirados de caprinos, foram encontrados 197 CI, 132 CII, 92 CIII e 64 GI, 70 GII, 91 GIII, 123 D, 74 A. Os dados obtidos demonstram não haver correlações significativas entre qualidade dos oócitos e diâmetro dos folículos $(\mathrm{P}>0,05)$. Nas condições em que se desenvolveu este estudo, os resultados permitem concluir que o diâmetro folicular não exerce influência sobre a qualidade do complexo cumulus-oócito (COC) recuperado de ovários de fêmeas ovinas e caprinas.

PALAVRAS-CHAVES: Células cumulus, folículo aspirado, qualidade oocitária.

\section{ABSTRACT}

\section{EFFECT OF FOLLICULAR DIAMETER ON THE QUALITY OF OOCYTE OBTAINED FROM OVARIES OF SHEEPS (Ovis aries) AND GOATS (Capra hircus)}

This study aimed to evaluate the influence of follicular diameter on the quality of oocytes from ovaries of female sheep and goats. The ovaries of sheep (156 units) and goats (105 units) from slaughterhouse were used. The follicles were measured, aspirated and divided into three classes of follicular diameter: class $1(\mathrm{CI})-2$ to $3 \mathrm{~mm}$, class 2 (CII) -4 to $5 \mathrm{~mm}$ and class 3 (CIII) $-6 \mathrm{~mm}$. The recovered oocytes were evaluated and classified according to morphological aspect in 5 quality groups: grade 1 (GI), grade 2 (GII), grade 3 (GIII), naked (N) and atresia (A). Of the 468 follicles aspirated from sheep, 327 CI, 84 CII, CIII 57 and 83 GI, 78 GII, GIII 95, 119 N, 93 A were found, and from the 422 follicles aspirated from goats $197 \mathrm{CI}, 132 \mathrm{CII}, 92 \mathrm{CIII}$ and $64 \mathrm{GI}$, 70 GII, 91 GIII, 123 N, 74 A were found. The data did not show significant correlation between quality of oocytes and diameter of follicles $(\mathrm{P}>0.05)$. Under the conditions observed in this study it was concluded that the follicular diameter has no influence on the quality of cumulus-oocyte complex (COC) recovered from the ovaries of female sheep and goats.

KEYWORDS: Aspirated follicle, cumulus cells, oocyte quality. 


\section{INTRODUÇÃO}

Nos meados da década de 1980 foram obtidos os primeiros produtos oriundos da fecundação in vitro (FIV) de oócitos ovinos (CHENG et al., 1986) e de oócitos caprinos maturados in vitro (HANADA, 1985). Alguns anos mais tarde, foi relatado o nascimento do primeiro cordeiro oriundo da realização de todas as etapas in vitro, isto é, desde a maturação dos oócitos até o desenvolvimento de mórulas (CROZET et al., 1987).

A biotécnica de produção in vitro de embriões (PIVE) de pequenos ruminantes obteve avanços e foi rapidamente incorporada à reprodução destas espécies. No entanto, a taxa de desenvolvimento embrionário ainda é, em média, $10 \%$ a $15 \%$ dos oócitos ovinos (BERNARDI, 2005) e 5\% a 10\% dos oócitos caprinos (GIBBONS et al., 2008) maturados, que chegam até o estádio de blastocisto.

A colheita de oócitos por Ovum Pick-Up guiada por laparoscopia (LOPU) tem sido usada com sucesso na espécie ovina (BALDASSARRE et al., 1996) e na espécie caprina (TERVIT, 1996). A LOPU em ovelhas e cabras não tratadas com hormônios propicia a recuperação de, aproximadamente, quatro oócitos por fêmea ovina e seis oócitos por fêmeas caprinas (RUBIANES \& MENCHACA, 2003). No caso de fêmeas estimuladas com FSH ou FSH/LH, o número de oócitos obtido por sessão de aspiração foi de dez a onze para as duas espécies (PTAK et al., 1999).

Os cientistas tentam entender o sistema reprodutivo de fêmeas domésticas através da manipulação do desenvolvimento folicular, procurando produzir maior número de oócitos competentes para a fecundação (BLONDIN et al., 2002). Numerosas pesquisas têm estudado as diferentes características foliculares, como competência para o crescimento, tamanho folicular, folículos saudáveis, perfil hormonal e status ovariano (CASTILHO \& GARCIA, 2005).

Durante o período interovulatório em pequenos ruminantes, o crescimento folicular ocorre em padrões de ondas, tanto na estação de acasalamentos (EVANS et al., 2000; VINOLES et al., 2002) como na estação de anestro (BARTLESWKI et al., 1998) e no período de transição entre estas duas estações (RAVINDRA \& RAWLINGS, 1997). O número de ondas foliculares por ciclo varia entre duas e quatro em ovinos (VINOLES et al., 2002) e quatro e seis em caprinos (BALDASSARRE et al., 2002), com altas variações individuais, sendo mais frequentes três ondas foliculares em ovelhas e cinco em cabras. Dessa forma, a maioria das aspirações foliculares é realizada em estádio aleatório do ciclo estral, em diferentes fases de crescimento folicular e sujeito a diferentes concentrações hormonais, o que afeta a qualidade dos oócitos obtidos (GIBBONS et al., 2008).

A influência do tamanho folicular na qualidade e competência oocitária para o desenvolvimento in vitro tem sido exaustivamente estudada em bovinos, e esses estudos indicam que folículos maiores geram oócitos com melhor competência (PAVLOK et al., 1992; LONERGAN et al., 1994; CAROLAN et al., 1996; HENDRIKSEN et al., 2000; SENEDA et al., 2001). No entanto, ainda não há um consenso entre os trabalhos em bovinos e, principalmente, quando são estudadas ovelhas (TERVIT et al., 1972) e cabras (IZQUIERDO et al., 1999). O presente estudo teve como objetivo avaliar a influência do diâmetro folicular sobre a qualidade dos oócitos obtidos de ovários de fêmeas ovinas e caprinas abatidas em matadouros.

\section{MATERIAL E MÉTODOS}

Os ovários utilizados foram obtidos de fêmeas ovinas e caprinas abatidas no abatedouro Suimax, localizado na cidade de Igarassu, Região Metropolitana da Cidade do Recife. Colocaram-se os ovários em recipiente térmico contendo solução salina a $0,9 \%$ aquecida a $30^{\circ} \mathrm{C}$ e acrescida $30 \mu \mathrm{g} / \mathrm{mL}$ de sulfato de gentamicina. Imediatamente foram transportados ao Laboratório de Biotécnica da Reprodução do Departamento de Medicina Veterinária da Universidade Federal Rural de Pernambuco, em tempo não superior a uma hora, após o abate.

No laboratório, os ovários foram lavados em solução salina, mantidos em banho-maria a $37^{\circ} \mathrm{C}$ e todos os folículos visíveis foram mensurados com paquímetro e divididos em três classes de diâmetro folicular: classe I (CI) - medindo de 2 até $3 \mathrm{~mm}$; classe II (CII) - medindo $4 \mathrm{~mm}$ até $5 \mathrm{~mm}$ e classe III $(\mathrm{CIII}) \geq 6 \mathrm{~mm}$. Após ser realizada essa classificação, procedeu-se à aspiração dos oócitos com o auxílio de seringas de $5 \mathrm{~mL}$ e agulhas 19G1/2.

Os oócitos recuperados foram colocados em 
placas de Petri com DMPBS (Nutricell) e líquido folicular, para serem observados e selecionados em lupa estereomicroscópica (Nikon, SMZ800), com objetiva de 4x. Nesta seleção, observou-se o aspecto morfológico do complexo cumulus-oócito (COC), segundo LONERGAN et al. (1994), em cinco grupos de qualidade. O grau I (GI) apresenta cumulus compacto, contendo mais de três camadas de células; o grau II (GII), cumulus compacto parcialmente presente em volta do oócito ou rodeando completamente o oócito, com menos de três camadas celulares; o grau III (GIII), cumulus presente, com apenas uma camada de célula; desnudo (D), ausência de camada de célula do cumulus, e atrésicos (A), células do cumulus em regressão.

Para fins de comparação, os oócitos foram classificados em escores segundo sua qualidade em oócitos atrésicos escore 0 , oócitos desnudos escore 1, oócitos grau I escore 2, oócitos grau II escore 3 e oócitos grau III escore 4. Para correlacionar os escores de qualidade oocitária com o tamanho folicular, empregou-se o teste não paramétrico de Spearman.

\section{RESULTADOS}

Foram aspirados 468 folículos ovinos, sendo 327 classificados como classe I, 83 classe II e 57 classe III. Quanto ao aspecto morfológico dos COCs, foram 83 grau I, 78 grau II, 95 grau III, 119 desnudos e 93 atrésicos. Não se observou correlação significativa $(\mathrm{P}>0,05)$ entre qualidade dos oócitos e tamanho dos folículos $(\mathrm{r}=0,02018)$. Os resultados obtidos para os diferentes tamanhos dos oócitos ovinos avaliados e as respectivas classificações sobre a qualidade estão apresentados na Tabela 1.

Os resultados obtidos para os diferentes tamanhos de folículos avaliados e as respectivas classificações sobre a qualidade dos 422 folículos de fêmeas caprinas aspirados estão apresentados na Tabela 2. Desses, 197 foram classificados como Classe I, 132 Classe II e 92 Classe III. Quanto ao aspecto morfológico dos COCs observados, 64 grau I, 70 grau II, 91 grau III, 123 desnudos e 74 atrésicos. Não foi observada correlação significativa $(\mathrm{P}>0,05)$ entre qualidade dos oócitos e tamanho dos folículos $(\mathrm{r}=0,02421)$.

TABELA 1. Número e porcentagem de oócitos obtidos de folículos aspirados de ovários de fêmeas ovinas abatidas nos diâmetros CI (2-3 mm), CII (4-5 mm) e CIII (6 mm)

\begin{tabular}{ccccccc}
\hline Classes & $\begin{array}{c}\text { Grau I } \\
\mathrm{n}(\%)\end{array}$ & $\begin{array}{c}\text { Grau II } \\
\mathrm{n}(\%)\end{array}$ & $\begin{array}{c}\text { Grau III } \\
\mathrm{n}(\%)\end{array}$ & $\begin{array}{c}\text { Desnudo } \\
\mathrm{n}(\%)\end{array}$ & $\begin{array}{c}\text { Atrésico } \\
\mathrm{n}(\%)\end{array}$ & $\begin{array}{c}\text { Total } \\
\mathrm{n}(\%)\end{array}$ \\
\hline CI & $58(17,7)$ & $54(16,6)$ & $61(18,7)$ & $92(28,1)$ & $62(18,9)$ & $327(69,8)$ \\
CII & $10(11,9)$ & $12(14,3)$ & $21(25,0)$ & $14(16,7)$ & $27(32,1)$ & $84(17,9)$ \\
CIII & $15(26,3)$ & $12(21,0)$ & $13(22,8)$ & $13(22,8)$ & $04(7,0)$ & $57(12,2)$ \\
Total & $83(17,7)$ & $78(16,6)$ & $95(20,3)$ & $119(25,4)$ & $93(19,9)$ & $468(100)$ \\
\hline
\end{tabular}

$\mathrm{n}=468$ (número total de oócitos ovinos)

TABELA 2. Número e porcentagem de oócitos obtidos de folículos aspirados de ovários de fêmeas caprinas abatidas nos diâmetros CI (2-3 mm), CII (4-5 mm) e CIII (6 mm)

\begin{tabular}{|c|c|c|c|c|c|c|}
\hline Classes & $\begin{array}{c}\text { Grau I } \\
\mathrm{n}(\%)\end{array}$ & $\begin{array}{c}\text { Grau II } \\
\text { n (\%) }\end{array}$ & $\begin{array}{c}\text { Grau III } \\
\text { n }(\%)\end{array}$ & $\begin{array}{c}\text { Desnudo } \\
\mathrm{n}(\%)\end{array}$ & $\begin{array}{c}\text { Atrésico } \\
\text { n }(\%)\end{array}$ & $\begin{array}{l}\text { Total } \\
\mathrm{n}(\%)\end{array}$ \\
\hline CI & $18(9,1)$ & $30(15,2)$ & $37(18,7)$ & $67(34,1)$ & $45(22,8)$ & $197(46,6)$ \\
\hline CII & $29(21,9)$ & $22(16,6)$ & $29(21,9)$ & $34(25,7)$ & $18(13,7)$ & $132(31,3)$ \\
\hline CIII & $17(18,3)$ & $18(19,3)$ & $25(25,8)$ & $22(23,6)$ & $11(11,8)$ & $93(22,1)$ \\
\hline Total & $64(15,1)$ & $70(16,6)$ & $91(21,5)$ & $123(29,1)$ & $74(17,5)$ & $422(100)$ \\
\hline
\end{tabular}

$\mathrm{n}=422$ (número total de oócitos caprinos) 


\section{DISCUSSÃO}

A ausência de correlação entre o diâmetro folicular e a qualidade dos oócitos pode ser atribuída à dinâmica folicular característica dos pequenos ruminantes como citado por VINOLES et al. (2002). Dessa forma, em qualquer fase do ciclo estral existem folículos em crescimento, ou sofrendo atresia. A atresia dos folículos recrutados ocorre em função da diminuição da concentração plasmática de FSH (ADAMS et al.,1992), induzindo no folículo queda no estradiol intrafolicular (KRUIP \& DIELEMAN, 1985; KRUIP et al., 1994). Todas essas alterações hormonais induzem alterações na qualidade do oócito.

Segundo VASSENA et al. (2003), a qualidade do oócito é de suma importância nas biotecnologias de reprodução assistida. A presença das células do cumulus é benéfica para a obtenção de embriões após a FIV, pois oócitos desnudos têm uma taxa de clivagem baixa (FATEHI et al., 2005). FUKUI \& SAKUMA (1980) observaram que em bovinos não houve diferença na maturação in vitro em oócitos de diferentes qualidades, mas quando os oócitos eram recuperado de folículos de menor diâmetro $(\leq 4 \mathrm{~mm})$, possuíam qualidade superior aos de maior diâmetro (>4 mm).

BLONDIN \& SIRARD (1995) observaram em bovinos que a competência para o desenvolvimento embrionário in vitro somente foi afetada por altos níveis de atresia nas células do cumulus. Os oócitos com células do cumulus exibindo sinais médios de atresia tiveram taxas maiores de desenvolvimento embrionário que oócitos sem sinais de atresia.

A condição fisiológica da doadora, como peso, raça, idade e variação individual, tem merecido atenção em diversos estudos. RUBIANES \& MENCHACA (2003), trabalhando com ovários de cabras de matadouro, não encontraram diferenças na qualidade dos oócitos entre animais jovens (a partir de dezoito meses) e senis (até dezessete anos), embora tenham notado redução na produção de gametas nos animais mais velhos.

CAROLAN et al. (1996) não observaram influência do diâmetro folicular sobre a maturação do oócito em bovinos. Resultado similar foi observado por SENEDA et al. (2001), ao estudarem oócitos bovinos oriundos de folículos pequenos $(<4 \mathrm{~mm})$ e grandes (> $4 \mathrm{~mm}$ ) aspirados in vivo.
Por outro lado, em bovinos, os oócitos contidos em folículos maiores possuem melhor competência para o desenvolvimento in vitro (PAVLOK et al., 1992; LONERGAN et al., 1994; HENDRIKSEN et al., 2000). RAUBER et al. (2003) citaram que, provavelmente, as substâncias estimuladoras estejam presentes nos folículos grandes e médios e ausentes ou presentes em menor concentração em folículos pequenos. Já CASTILHO et al. (2007), estudando o diâmetro e a fase de desenvolvimento folicular em bovinos da raça Nelore, concluíram que o desenvolvimento folicular influencia a competência oocitária para o desenvolvimento in vitro, observando que, nos estádios iniciais da onda folicular, a produção de blastocisto foi maior em oócitos de folículos pequenos e, com o avanço da onda, a produção de blastocistos foi maior em oócitos obtidos de folículos maiores.

FIGUEIREDO et al. (1997) concluíram que, além do fator individual, outro aspecto de ordem biológica a ser considerado como possível variável na FIV é a interação folículo-oócito. RHODES et al. (1997), DAYAN et al. (2000) e ALVES et al. (2006), estudando a competência do oócito com aspectos indiretamente ligados ao diâmetro folicular, a relação entre componentes do fluido e diâmetro folicular, a interação entre diâmetro do folículo e qualidade do cumulus oophorus, respectivamente, afirmaram que há uma escassez na literatura quanto à influência do diâmetro do folículo sobre a qualidade e competência do oócito para o desenvolvimento, quando o oócito é obtido pela técnica de Ovum Pick-Up guiada por laparoscopia (LOPU).

\section{CONCLUSÃO}

Os resultados indicam que não houve influência do diâmetro folicular sobre a qualidade dos complexos cumulus-oócitos (COC's) recuperados de ovários de fêmeas ovinas e caprinas oriundas de abatedouros, em fase aleatória do ciclo estral.

\section{AGRADECIMENTOS}

À Empresa Suimax, por disponibilizar os animais necessários à execução deste experimento. 


\section{REFERÊNCIAS}

ADAMS, G. P.; MATTERI, R. L.; KASTELIC, J. P.; KO, J. C. H.; GINTHER, O. J. Association between surges of follicle-stimulating hormone and the emergence of follicular waves in heifers. Journal of Reproduction and Fertility, v. 94, p. 177-188, 1992.

ALVES, D. F.; RAUBER, L. P.; RUBIN, F. B. Desenvolvimento embrionário in vitro de oócitos bovinos mantidos em líquido folicular ou TCM-hepes. Brazilian Journal Veterinarian Research and Animal Science, v. 40, n. 4, p. 279-286, 2006.

BALDASSARRE, H.; FURNUS, C. C.; DE MATOS, D. G.; PESSI, $\mathrm{H}$. In vitro production of sheep embryos using laparoscopic folliculocentesis: alternative gonadotrophin treatments for stimulation of oocyte donors. Theriogenology, v. 45, p. 707-717, 1996.

BALDASSARRE, H.; WANG, B.; KAFIDI, N.; KEEFER, C.; LAZARIS, A.; KARATZAS, C. N. Advances in the production and propagation of transgenic goats using laparoscopic ovum pick-up and in vitro embryo production technologies. Theriogenology, v. 57, p. 275-284, 2002.

BARTLEWSKI, P. M.; BEARD, A. P.; COOK, S. J.; RAWLINGS, N. C. Ovarian follicular dynamics during anoestrus in ewes. Journal of Reproduction and Fertility, v. 113, p. 275-285, 1998.

BERNARDI, M. L. Produção in vitro de embriões ovinos. Acta Scientiae Veterinarian, v. 33, n. 1, p. 1-16, 2005.

BLONDIN, P.; BOUSQUET, D.; TWAGIRAMUGU, H.; BARNERS, F.; SIRARD, M.A. Manipulation of follicular development to produce developmentally competent bovine oocytes. Biologic of Reproduction, v. 66, p. 38-43, 2002.

BLONDIN, P.; SIRARD, M. A. Oocyte and follicular morphology as determining characteristics for developmental competence in sheep oocytes. Journal of Reproduction and Fertility, v. 62, p. 575-582, 1995.

CAROLAN, C.; LONERGAN, P.; MONGET, P.; MONNIAUX, D.; MERMILLOD, P. Effect of follicle size and quality on the ability of follicular fluid to support cytoplasmatic maturation of bovine oocytes. Molecule Reproduction Development, v. 43, p. 477-483, 1996.

CASTILHO, C.; GARCIA, J. M. Divergência no crescimento folicular: efeito na competência oocitária para o desenvolvimento in vitro de embriões - revisão. Archives of Veterinary Science, v. 10, n. 3 , p. $17-23,2005$.

CASTILHO, C.; ASSIS, G. S.; GARCIA, J. M. Influência do diâmetro e da fase folicular sobre a competência in vitro de oócitos obtidos de novilhas da raça Nelore. Arquivo Brasileiro de Medicina Veterinária e Zootecnia, v. 59, n. 2, p. 288-294, 2007.
CHENG, W. T. K.; MOOR, R. M.; POLGE, C. In vitro fertilization of pig and sheep oocytes matured in vivo and in vitro. Theriogenology, v. 25, p. 146, 1986.

CROZET, N.; HUNEAU, D.; DE SMEDT, V.; THÉRON, M-C.; SZÖLLÖSI, D.; TORRÈS, S.; SÉVELLEC, C. In vitro fertilization with normal development in the sheep. Gamete Research, v. 16, p. 159-170, 1987.

DAYAN, A.; WATANABE, M. R.; WATANABE, Y. F. Fatores que interferem na produção comercial de embriões. Arquivo da Faculdade de Veterinária da UFRGS, v. 20, n. 2, p. 181-185, 2000.

EVANS, A. C. O.; DUFFY, P.; HYNES, N.; BOLAND, M. P. Waves of follicle development during the estrous cycle in sheep. Theriogenology, v. 53, n. 3, p. 699-716, 2000.

FATEHI, A. N.; ROELEN, B. A. J.; COLENBRANDER, B.; SCHOEVERS, E. J.; GADELLA, B. M.; BEVERS, M. M.; VAN DEN HURK, $\mathrm{R}$. Presence of cumulus cells during in vitro fertilization protects the bovine oocyte against oxidative stress and improves first cleavage but does not affect further development. Zygote, v. 13, n. 2, p. 177-185, 2005.

FIGUEIREDO, R. A.; BARROS, C. M.; PINHEIRO, O. L.; SOLER, J. M. P. Ovarian follicular dynamics in Nelore Breed (Bos indicus). Theriogenology, v. 47, p. 1489-1505, 1997.

FUKUI, Y.; SAKUMA, Y. Maturation of bovine oocytes cultured in vitro: relation to ovarian activity, follicular size and the presence or absence of cumulus cells. Biology of Reproduction, v. 22, p. 669-673, 1980

GIBBONS, A.; PEREYRA BONNET, F.; CUETO, M. I.; SALAMONE, D.; CATALA, M. Colheita de oócitos guiadas por laparoscopia em caprinos e ovinos. Acta Scientiae Veterinariae, v. 36, Supl., p. 223-230, 2008.

HANADA, A. In vitro fertilization in goats. Journal of Animal Reproduction, v. 31, p. 21-26, 1985.

HENDRIKSEN, P. J. M.; VOS, P. L. A. M.; STEENWEG, W. N. M.; BEVERS, M. M.; DIELEMAN, S. J. Bovine follicular development and its effect on the in vitro competence of oocytes. Theriogenology, v. 53, n. 1, p. 11-20, 2000.

IZQUIERDO, D.; VILLAMEDIANA, P.; PARAMIO, M. T. Effect of culture medium on embryo development on pre-pubertal goat IVM-IVF oocytes. Theriogenology, v. 52, p. 847-861, 1999.

KRUIP, T. A.; DIELEMAN, S. J. Steroid hormone concentrations in the fluid of bovine follicles relative to size, quality and stage of the estrus cycle. Theriogenology, v. 24, p. 395-408, 1985.

KRUIP, T.A.; BONI, R. Potential use of ovum pick-up for embryo production and breeding in cattle. Theriogenology, v. 42, p. 675-684, 1994. 
LONERGAN, P.; MONAGHAN, P.; RIZOS, D. Effect of follicle size on bovine oocyte quality and development competence following maturation, fertilization and culture in vitro. Molecule Reproduction Development, v. 37, p. 48 -53, 1994.

PAVLOK, A.; LUCANS-HAHN, A.; NIEMANN, H. Fertilization and developmental competence of bovine oocytes derived from different categories of antral follicles. Molecule Reproduction Development, v. 31, p. 63-67, 1992.

PTAK, G.; DATTENA, M.; LOI, P.; TISCHNER, M.; CAPPAI, P. Ovum pick-up in sheep: efficiency of in vitro embryo production, vitrification and birth of offspring. Theriogenology, v. 52, p. 1105-1114, 1999.

RAUBER, L. P.; ALVES, D. F.; FIGUEIRÓ, G. M.; BRUM, D. S.; HILGERT, T. F.; BERNARDI, M. L.; SILVA, C. A. M.; RUBIN, M. I. B. Desenvolvimento embrionário de oócitos bovinos mantidos em fluido folicular bovino de folículos de diferentes diâmetros. Brazilian Journal Veterinary Research and Animal Science, v. 40, p. 169-177, 2003.

RAVINDRA, J. P.; RAWLINGS, N. C. Ovarian follicular dynamics in ewes during the transition from anoestrus to the breeding season. Journal of Reproduction and Fertility, v. 110, p. 279289, 1997.

RHODES, F. M.; PETERSON, A. J.; JOLLY, P. D.; MCMILLAN, W. H.; DONNISON, M.; LEDGARD, A.; PARTON, G.; HALL,
D. R. Bovine ovarian follicle and oocyte characteristics after emergence of the first follicular wave. Theriogenology, v. 47, n. 1, p. 149, 1997.

RUBIANES, E.; MENCHACA, A. The pattern and manipulation of ovarian follicular growth in goats. Animals Reproduction Science, v. 78, p. 271-287, 2003.

SENEDA, M. M.; ESPER, C. R.; GARCIA, J. M.; OLIVEIRA, J. A.; VANTINI, R. Relationship between follicle size and ultrasoundguided transvaginal oocyte recovery. Animals Reproduction Science, v. 67, p. 37-43, 2001.

TERVIT, H. R.; WHITTINGHAM, D. G.; ROWSON, L. E. A. Successful culture in vitro sheep and cattle ova. Journal of Reproduction and Fertility, v. 30, p. 493-497, 1972.

TERVIT, H. R. Laparoscopy/laparotomy oocyte recovery and juvenile breeding. Animal Reproduction Science, v. 42, p. 227238, 1996.

VASSENA, R.; MAPLETOFT, R. J.; ALLODI, S.; SINGH, J.;ADAMS, G. P. Developmental competence of bovine oocytes relative to follicular status. Theriogenology, v. 15, p. 923-932, 2003.

VIÑOLES, C.; FORSBERG, M.; BANCHERO, G.; RUBIANES, E. Ovarian follicular dynamics and endocrine profiles in Polwarth ewes with high and low body condition. Animal Science, v. 74, p. 539-545, 2002. 